\title{
Thermodynamics as a Tool for the Optimization of Drug Binding
}

\author{
Ruth Matesanz, Benet Pera and J. Fernando Díaz \\ Centro de Investigaciones Biológicas (C.S.I.C.) \\ Spain
}

\section{Introduction}

A non-covalent interaction is a kind of chemical bond, typically between macromolecules, that involves dispersed variations of electromagnetic interactions (Alberts et al. 1994; Connors \& Mecozzi 2010). Non-covalent interactions are individually weak as compared with covalent bonds, but their net strength is higher than the sum of that of the individual interactions. There are few drugs that bind irreversibly to their targets, in pharmacology, most drugs establish non-covalent interactions with their target molecules (usually proteins).

From a chemical point of view, the affinity constant $\left(\mathrm{K}_{\mathrm{a}}\right)$ is a very useful measurement for the study of binding reactions as it provides much information about the mechanism. In many cases some chemical or physical properties of ligand or target change with the interaction between them, these changes might help to measure binding constants. It is important to establish the stoichiometry of the complex to be sure that the constants are accurately calculated. From the affinity constants measured it is possible to calculate the standard thermodynamic quantities for the binding reaction: free-energy $(\Delta \mathrm{G})$, enthalpy $(\Delta \mathrm{H})$ and entropy $(\Delta \mathrm{S})$.

Our group has already demonstrated that, in some cases, binding affinity measurements are very helpful for the optimization of ligand binding as it can be determined the contribution of every single chemical modification of the ligand to the binding affinity (Buey et al. 2004; Matesanz et al. 2008)

One of the objectives of drug development is the search of new or modified compounds with improved properties such as better potency, higher selectivity, better pharmacokinetics or superior drug resistance profiles. An important goal in this objective is the optimization of drugs binding affinity towards their targets, as binding affinity is directly related to potency (Ruben et al. 2006). Moreover, it has been shown that extremely high affinity drugs reflect as well changes in other properties like selectivity (Ohtaka et al. 2004; Ohtaka \& Freire 2005) or resistance overcoming ability (Matesanz et al. 2008).

Examples of the importance of ligand affinity in drug optimization can be observed in the development of HIV-1 protease inhibitors and statins (cholesterol lowering drugs) over the years as remarked in (Freire 2008).

In this chapter we will study the nature of non-covalent interations and the concept of binding constant for these interactions. Examples of methodologies to measure binding constants of small ligands to macromolecules will be introduced and we will emphasize the 
need to determine the stoichiometry of the studied system to calculate accurately the constants. Once the thermodynamic concepts were introduced, we will show the use of these kind of studies for the optimization of drug binding to its target. We will detail the role of single chemical modifications in the molecule of study to modulate its binding affinity, and the way to quantify these changes. We will finally further discuss how the selection of the best sustituents can result in the optimization of binding.

\section{Non-covalent interactions}

Non-covalent interactions are chemical bonds that do not involve sharing of electron pairs between orbitals of different atoms, there are no orbital overlapping in these interactions which have an electrostatic nature and are not highly directional. Covalent bonds are generally shorter than $2 \AA$ while the non-covalent ones are within the range of several angstroms. Another difference between these two types of bonds is the energy released in its formation, non-covalent interactions are weaker, with energies below $40 \mathrm{~kJ} / \mathrm{mol}$ whereas covalent bonds energies range $80-800 \mathrm{~kJ} / \mathrm{mol}$.

These weak interactions have important roles in the binding of macromolecules with each other and with other molecules in the cell, in the mainteinance of the three dimensional structure of large macromolecules such as proteins or nucleic acids (e.g. DNA double helix) and they are the forces found in the majority of the drug-proteins interactions in pharmacology.

\subsection{Types of non-covalent interactions}

There are four commonly mentioned fundamental non-covalent interaction types including ionic interactions, hydrogen bonds, hydrophobic interactions and van der Waals forces (dispersion attractions, dipole-dipole and dipole-induced dipole interactions). All these weak interactions must work together to have significant effects. Their combined bond effect is greater than the sum of the individual ones. The free energy of multiple bonds between two molecules is different than the sum of the enthalpies of each bond due to entropic effects.

\subsection{1 lonic interactions}

Ionic bonds result from the electrostatic attraction between two ionized groups of opposite charge such as carboxyl (-COO-) and amino $\left(-\mathrm{NH}_{3}{ }^{+}\right)$. These ionic interactions are directly proportional to the product of the interacting charges and inversely proportional to the dielectric constant of the medium and the distance separating the charges. This relationship is defined by Coulomb's law:

$$
\mathrm{E}=\frac{\mathrm{kq}_{1} \mathrm{q}_{2}}{\mathrm{Dr}}
$$

where $\mathrm{E}$ is the energy, $\mathrm{q}_{1}$ and $\mathrm{q}_{2}$ are the charges of two atoms, $\mathrm{r}$ is the distance between them, $\mathrm{D}$ is the dielectric constant, and $\mathrm{k}$ is a proportionality constant. A charged group on a molecule can attract an oppositely charged group from another molecule. By contract, an attractive interaction has a negative energy. The dielectric constant is important for the medium. In water, these bonds are very weak as the dielectric constant is much higher $(D=80)$ than in vacuum $(D=1)$. As an example, the electrostatic interaction between two atoms bearing single opposite charges separated by $3 \AA$ in water has an energy of $5.9 \mathrm{~kJ} / \mathrm{mol}$ $(\mathrm{k}=1389 \mathrm{~kJ} / \mathrm{mol})$. 


\subsubsection{Van der Waals forces}

Van der Waals forces are short range attractive forces between chemical groups in contact. The forces are caused by slight charge displacements. The distribution of electronic charge around an atom changes with time. At any moment, the charge distribution is not perfectly symmetric. This transient asymmetry in the electronic charge around an atom induces a complementary asymmetry in the electron distribution around its neighboring atoms. These induced dipole effects give rise to the so called van der Waals interactions, also known as dispersion forces. The attraction between two atoms increases as they come closer to each other, until they are separated by the so called van der Waals contact distance. At a shorter distance, very strong repulsive forces become dominant because the outer electron clouds overlap. The van der Waals radius of an atom is defined where the net force between two atoms is zero. The van der Waals potential is then best described as a balance between attraction and repulsion.

Van der Waals forces are non-directional. Energies associated with them are quite small; typical interactions contribute from 2 to $4 \mathrm{~kJ} / \mathrm{mol}$ per atom pair. However, when the surfaces of two large molecules come together, a large number of atoms are in van der Waals contact, and the net effect, summed over many atom pairs, can be substantial.

\subsubsection{Hydrogen bonds}

A hydrogen bond is an interaction between a proton donor group (a hydrogen atom covalently bound to an electronegative atom -e.g. F, O, N, S-) and a proton acceptor atom (another electronegative atom). It is a very important interaction responsible for the structure and properties of water, as well as the structure and properties of biological macromolecules (e.g. hydrogen bonds are responsible of specific base-pair formation in the DNA double helix).

Hydrogen bonds are fundamentally electrostatic interactions. The relatively electronegative atom to which the hydrogen atom is covalently bonded pulls electron density away from the hydrogen atom so that it develops a partial positive charge $\left(\delta^{+}\right)$. Thus, it can interact with an atom having a partial negative charge $(\delta-)$ through an electrostatic interaction. However, this interaction is more than just an ionic or dipole-dipole interaction between the donor and the acceptor groups. Here, the distance between the hydrogen and acceptor atoms is less than the sum of their respective van der Waals radii.

Hydrogen bonds are directional toward the electronegative atom. The strongest hydrogen bonds have a tendency to be approximately straight, such that the proton donor group, the hydrogen atom, and the acceptor atom lie along a straight line, with significant weakening of the interaction if they are not colinear. They are somewhat longer than are covalent bonds. Hydrogen bonds are constantly being made and remade. Their half-life is about 10 seconds. These bonds have only $5 \%$ or so of the strength of covalent bonds. They have energies of $5-15 \mathrm{~kJ} / \mathrm{mol}$ compared with approximately $420 \mathrm{~kJ} / \mathrm{mol}$ for a carbon-hydrogen covalent bond. However, when many hydrogen bonds can form between two molecules (or parts of the same molecule), the resulting union can be sufficiently strong as to be quite stable. Examples of multiple hydrogen bonds are widely found in biological systems, they hold secondary structures of polypeptides, help in binding of enzymes to their substrate or antibodies to their antigen, help also transcription factors bind to each other or to DNA.

\subsubsection{Hydrophobic interactions}

Hydrophobic interactions result when non-polar molecules are in a polar solvent (e.g. water). The non-polar molecules group together to exclude water so that they minimize the 
surface area in contact with the polar solvent. Unlike the non-covalent interactions mentioned above, which are pairwise interactions between atoms or parts of molecules, the nature of the hydrophobic interaction is very different. It involves a considerable number of (water) molecules, and does not arise from a direct force between the non-polar molecules. Nonpolar molecules are not good acceptors of hydrogen bonds. When a non-polar molecule is placed in water, the hydrogen bonding network of water is disrupted. Water molecules must reorganize around the solute and make a kind of cage, similar to the structure of water in ice, in order to gain back the broken hydrogen bonds. This reorganization results in a considerable loss in the configurational entropy of water and therefore, in an increase in the free energy. If there are more than one such non-polar molecules, the configuration in which they are clustered together is preferred because now the hydrogen bonding network of water is disrupted in just one (albeit bigger) pocket, rather than in several small pockets. Therefore, the entropy of water is larger when the non-polar molecules are clustered together, leading to a decrease in the free energy.

Hydrophobic interactions have strengths comparable in energy to hydrogen bonds.

\section{Binding constants}

Most drugs have a non-covalent binding to their targets, thus these interactions are of great importance for our studies. Measurements of equilibrium constants, their dependence with temperature, the determination of stoichiometry, provide main information on the mechanism of the chemical process involved. The basic process can be taken out of the association of ligand (or ligands) to its target. The binding reaction can be writen as follows:

$$
\mathrm{mP}+\mathrm{nL} \leftrightarrow \mathrm{P}_{\mathrm{m}} \mathrm{L}_{\mathrm{n}}
$$

Regardless of mechanism, every reversible reaction reaches an equilibrium distribution of reactants and products. At some point the rates of the opposing reactions (association and dissociation in our case) become equal and there would no longer be any change in the concentration of the molecules implied.

$$
\begin{aligned}
& v_{\text {ass }}=k_{\text {ass }}[P]^{m}[L]^{n} \\
& v_{\text {diss }}=k_{\text {diss }}\left[P_{m} L_{n}\right]
\end{aligned}
$$

Under these conditions $\left(\mathrm{v}_{\text {ass }}=\mathrm{v}_{\text {diss }}\right)$ :

$$
\frac{\mathrm{k}_{\text {ass }}}{\mathrm{k}_{\text {diss }}}=\frac{\left[\mathrm{P}_{\mathrm{m}} \mathrm{L}_{\mathrm{n}}\right]}{[\mathrm{P}]^{\mathrm{m}}[\mathrm{L}]^{\mathrm{n}}}=\mathrm{K}_{\mathrm{a}}
$$

that will be the equilibrium association constant assuming that activities are equal to concentrations.

In this section we will discuss the cases for one single site in the target, multiple sites with same affinities and multiple sites with different affinities.

\subsection{One-site binding}

In the simplest case, where there is only one site per target molecule, $\mathrm{n}$ and $\mathrm{m}$ are 1 . It is possible to define the fraction of occupied binding sites (v) as: 


$$
\mathrm{v}=\frac{\mathrm{L}_{\text {bound }}}{\mathrm{P}_{\text {total }}}=\frac{[\mathrm{PL}]}{[\mathrm{PL}]+[\mathrm{P}]}
$$

Determining $\mathrm{v}$ is often easy in spectrophotometric manipulation as will be discussed later. Given $v$, equation 5 can then be solved for [PL] and the answer substituted into equation 6 to obtain the quantitative 1:1 stoichiometric model:

$$
\mathrm{v}=\frac{\mathrm{K}_{\mathrm{a}}[\mathrm{L}]}{1+\mathrm{K}_{\mathrm{a}}[\mathrm{L}]}
$$

This equation is the 1:1 binding isotherm also known as the Langmuir isotherm or the "direct" plot. Its functional form is a rectangular hyperbola whose midpoint will yield $\mathrm{K}_{\mathrm{a}}$. Chemical interpretation of 1:1 binding is that the target $\mathrm{P}$ has a single "binding site", as has the ligand L; and when the complex PL forms, no further sites are available for the binding of any additional ligand. To test the 1:1 stoichoimetry equation 7 may be rearrange into a linear plotting form. Since $v$ is the bound fraction, then 1-v is the free one: $(1-v)=$ $1 /\left(1+\mathrm{K}_{\mathrm{a}}[\mathrm{L}]\right)$. Thus $\mathrm{v} /(1-\mathrm{v})=\mathrm{K}_{\mathrm{a}}[\mathrm{L}]$, and:

$$
\log \frac{\mathrm{v}}{1-\mathrm{v}}=\log [\mathrm{L}]+\log \mathrm{K}_{\mathrm{a}}
$$

This log-log plot should be linear with a slope of one if the stoichoimetry is really $1: 1$. This is called a Hill plot. Equation 7 can be also rearranged to three different non-logarithmic linear plotting forms. Taking simply the reciprocal of the equation yields the double-reciprocal plot (used by plotting $1 / \mathrm{v}$ against $1 /[\mathrm{L}]$ ):

$$
\frac{1}{v}=\frac{1}{K_{a}[L]}+1
$$

In spectroscopic studies this plot is commonly known as the Benesi-Hildebrand plot (Benesi \& Hildebrand 1949).

Another plot is that of $[\mathrm{L}] / \mathrm{v}$ against [L] which is expected to be linear:

$$
\frac{[\mathrm{L}]}{\mathrm{v}}=[\mathrm{L}]+\frac{1}{\mathrm{~K}_{\mathrm{a}}}
$$

And the third plotting of $\mathrm{v} /[\mathrm{L}]$ agains v, sometimes called Scatchard plot (Scatchard 1949):

$$
\frac{\mathrm{v}}{[\mathrm{L}]}=\mathrm{vK}_{\mathrm{a}}+\mathrm{K}_{\mathrm{a}}
$$

Linearity in all of these plots is a necessary condition if the 1:1 model is valid; and from the parameters of equations $\mathrm{K}_{\mathrm{a}}$ can be evaluated. Usually $\mathrm{v}$ is not measured directly but rather some experimental quantity related to it, so that the interpretation of the plots depends on the particular experimental methodology.

\subsection{Multi-site binding}

Most biological systems tend to have more than one binding site, that is the case of many systems of small molecules binding to proteins. In these cases we may consider that $n$ ligands may bind to a single target molecule. The average number of ligand molecules bound per target molecule (b) is defined as:

$$
\mathrm{b}=\frac{\mathrm{L}_{\text {total }}-[\mathrm{L}]}{\mathrm{P}_{\text {total }}}
$$


Assuming that all $\mathrm{n}$ binding sites in the target molecule are identical and independent, it is possible to establish:

$$
\mathrm{b}=\frac{\mathrm{nk}[\mathrm{L}]}{1+\mathrm{k}[\mathrm{L}]}
$$

where $\mathrm{k}$ is the constant for binding to a single site. According to this equation this system follows the hyperbolic function characteristic for the one-site binding model. To define the model $\mathrm{n}$ and $\mathrm{k}$ can be evaluated from a Scatchard plot. The affinity constant $\mathrm{k}$ is an average over all binding sites, it is in fact constant if all sites are truly identical and independent. A stepwise binding constant $\left(\mathrm{K}_{\mathrm{st}}\right)$ can be defined which would vary statistically depending on the number of target sites previously occupied. It means that for a target with $\mathrm{n}$ sites will be much easier for the first ligand added to find a binding site than it will be for each succesive ligand added. The first ligand would have $\mathrm{n}$ sites to choose while the nth one would have just one site to bind. The stepwise binding constant can be defined as:

$$
\mathrm{K}_{\mathrm{st}}=\frac{\text { number of free target sites }}{\text { number of bound sites }} \mathrm{k}=\frac{\mathrm{n}-\mathrm{b}+1}{\mathrm{~b}} \mathrm{k}
$$

It is interesting to notice that a deviation from linearity in the Scatchard plot (and to a lesser extent in the Benesi-Hildebrand) gives information on the nature of binding sites. A curved plot denotes that the binding sites are not identical and independent.

\subsection{Allosteric interactions}

Another common situation in biological systems is the cooperative effect, in that case several identical but dependent binding sites are found in the target molecule. It is important to define the effect of the binding of succesive ligands to the target to describe the system. An useful model for that issue is the Hill plot (Hill 1910). In this case the number of ligands bound per target molecule will be (take into account that the situation in this system for equation 2 is $m=1$ and $n \neq 1$ ):

$$
\mathrm{b}=\frac{\mathrm{n}\left[\mathrm{PL}_{\mathrm{n}}\right]}{\left[\mathrm{PL}_{\mathrm{n}}\right]+[\mathrm{P}]}
$$

if equation 5 is solved for $\left[\mathrm{PL}_{n}\right]$ and substitute into equation 15 , then:

$$
\mathrm{b}=\frac{\mathrm{nK}_{\mathrm{a}}[\mathrm{L}]^{\mathrm{n}}}{\mathrm{K}_{\mathrm{a}}[\mathrm{L}]^{\mathrm{n}}+1}
$$

This expression can be rewritten as:

$$
\frac{\mathrm{b}}{\mathrm{n}-\mathrm{b}}=\mathrm{K}_{\mathrm{a}}[\mathrm{L}]^{\mathrm{n}}
$$

Note that the fraction of sites bound, $v$ (see equation 6), is the number of sites occupied, $b$, divided by the number of sites available, $n$. Then equation 17 becomes:

$$
\frac{\mathrm{v}}{1-\mathrm{v}}=\mathrm{K}_{\mathrm{a}}[\mathrm{L}]^{\mathrm{n}}
$$

Equation 18 is known as the Hill equation. From the Hill equation we arrive at the Hill plot by taking logarithms at both sides:

$$
\log \frac{v}{1-v}=n_{H} \log [L]+\log K_{a}
$$


Plotting $\log (\mathrm{v} /(1-\mathrm{v}))$ against $\log [\mathrm{L}]$ will yield a straight line with slope $\mathrm{n}_{\mathrm{H}}$ (called the Hill coefficient). The Hill coefficient is a qualitative measure of the degree of cooperativity and it is experimentally less than the actual number of binding sites in the target molecule. When $n_{H}>1$, the system is said to be positively cooperative, while if $n_{H}<1$, it is said to be anticooperative. Positively cooperative binding means that once the first ligand is bound to its target molecule the affinity for the next ligand increases, on the other hand the affinity for subsequent ligand binding decreases in negatively cooperative (anti-cooperative) systems. In the case of $n_{H}=1$ a non-cooperative binding occurs, here ligand affinity is independent of whether another ligand is already bound or not.

Since equation 19 assumes that $n_{H}=n$, it does not described exactly the real situation. When a Hill plot is constructed over a wide range of ligand concentrations, the continuity of the plot is broken at the extremes concentrations. In fact, the slope at either end is approximately one. This phenomenon can be easily explained: when ligand concentration is either very low or very high, cooperativity does not exist. For low concentrations it is more probable for individual ligands to find a target molecule "empty" rather than to occupy succesive sites on a pre-bound molecule, thus single-binding is happening in this situation. At the other extreme, for high concentrations, every binding-site in the target molecule but one will be filled, thus we find again single-binding situation. The larger the number of sites in a single target molecule is, the wider range of concentrations the Hill plot will show cooperativity.

\section{Determination of binding constants}

As discuss above the binding constant provides important and interesting information about the system studied. We will present a few of the multiple experimental posibilities to measure this constant (further information could be found in the literature (Johnson et al. 1960; Connors 1987; Hirose 2001; Connors\&Mecozzi 2010; Pollard 2010)). It is essencial to keep in mind some crucial details to be sure to calculate the constants properly: it is important to control the temperature, to be sure that the system has reached the equilibrium and to use the correct equilibrium model. One common mistake that should be avoid is confuse the total and free concentrations in the equilibrium expression.

Different techniques are commonly used to study the binding of ligands to their targets. These techniques can be classified as calorimetry, spectroscopy and hydrodynamic methods. Hydrodynamic techniques are tipically separation methodologies such as different chromatographies, ultracentrifugation or equilibrium dialysis with which free ligand, free target and complex are physically separated from each other at equilibrium, thus concentrations of each can be measured. Spectroscopic methodologies include optical spectroscopy (e.g. absorbance, fluorescence), nuclear magnetic resonance or surface plasmon resonance. Calorimetry includes isothermal titration and differential scanning. Calorimetry and spectroscopy methods allow accurately determination of thermodynamics and kinetics of the binding, as well as can give information about the structure of binding sites.

Once the bound (or free) ligand concentration is measured, the binding proportion can be calculated. Other thermodynamic parameters can be calculated by varying ligand or target concentrations or the temperature of the system.

\subsection{Determination of stoichiometry. Continuous variation method.}

Since correct reaction stoichiometry is crucial for correct binding constant determination we will study how can it be evaluated. There are different methods of calculating the 
stoichoimetry: continuous variation method, slope ratio method, mole ratio method, being the first one, the continuous variation method the most popular. In order to determine the stoichiometry by this method the concentration of the produced complex (or any property proportional to it) is plotted versus the mole fraction ligand $\left([\mathrm{L}]_{\text {total }} /\left([\mathrm{P}]_{\text {total }}+[\mathrm{L}]_{\text {total }}\right)\right)$ over a number of tritation steps where the sum of $[\mathrm{P}]_{\text {total }}$ and $[\mathrm{L}]_{\text {total }}$ is kept constant $(\alpha)$ changing $[\mathrm{L}]_{\text {total }}$ from 0 to $a$. The maxima of this plot (known as Job's plot, (Job 1928; Ingham 1975)) indicates the stoichiometry of the binding reaction: $1: 1$ is indicated by a maximum at 0.5 since this value corresponds to $n /(n+m)$. For the understanding of the theoretical background of the method, it is important to remember equations 2 and 5; notice that:

$$
\begin{gathered}
{[\mathrm{P}]_{\text {total }}=[\mathrm{P}]+\mathrm{m}\left[\mathrm{P}_{\mathrm{m}} \mathrm{L}_{\mathrm{n}}\right]} \\
{[\mathrm{L}]_{\text {total }}=[\mathrm{L}]+\mathrm{n}\left[\mathrm{P}_{\mathrm{m}} \mathrm{L}_{\mathrm{n}}\right]} \\
\mathrm{a}=[\mathrm{L}]_{\text {total }}+[\mathrm{P}]_{\text {total }} \\
\mathrm{x}=\frac{[\mathrm{L}]_{\text {total }}}{[\mathrm{P}]_{\text {total }}+[\mathrm{L}]_{\text {total }}} \\
y=\left[\mathrm{P}_{\mathrm{m}} \mathrm{L}_{\mathrm{n}}\right]
\end{gathered}
$$

Substitution of $[\mathrm{P}]_{\text {total }}$ and $[\mathrm{L}]_{\text {total }}$ by the functions of $\mathrm{a}$ and $\mathrm{x}$ from equation 23 and 24 yields:

$$
\begin{gathered}
{[\mathrm{P}]_{\text {total }}=\alpha-a x} \\
{[\mathrm{~L}]_{\text {total }}=\alpha x}
\end{gathered}
$$

from equations $2,5,20,21,24,25,26$ :

$$
y=K_{a}(a-m y-a x)^{m}(a x-n y)^{n}
$$

Equation 27 is differentiated, and the $\mathrm{dy} / \mathrm{dx}$ substituted by zero to obtain the $\mathrm{x}$-coordinate at the maximum:

$$
x=\frac{n}{n+m}
$$

This equation shows the correlation between stoichiometry and the $x$-coordinate at the maximum in Job's plot. That's why a maximum at $x=0.5$ means a 1:1 stoichiometry $(n=m$ $=1$ ). In the case of 1:2 the maximum would be at $x=1 / 3$.

\subsection{Calorimetry}

Isothermal titration calorimetry (ITC) is a useful tool for the characterization of thermodynamics and kinetics of ligands binding to macromolecules. With this method the rate of heat flow induced by the change in the composition of the target solution by tritation of a ligand (or vice versa) is measured. This heat is proportional to the total amount of binding. Since the technique measures heat directly, it allows simultaneous determination of the stoichiometry $(\mathrm{n})$, the binding constant $\left(\mathrm{K}_{\mathrm{a}}\right)$ and the enthalpy $\left(\Delta \mathrm{H}^{0}\right)$ of binding. The free energy $\left(\Delta \mathrm{G}^{0}\right)$ and the entropy $\left(\Delta \mathrm{S}^{0}\right)$ are easily calculated from $\Delta \mathrm{H}^{0}$ and $\mathrm{K}_{\mathrm{a}}$. Note that the binding constant is related to the free energy by:

$$
\Delta \mathrm{G}^{0}=-\mathrm{RT} \ln \mathrm{K}_{\mathrm{a}}
$$


where $\mathrm{R}$ is the gas constant and $\mathrm{T}$ the absolute temperature. The free energy can be dissected into enthalpic and entropic components by:

$$
\Delta \mathrm{G}^{0}=\Delta \mathrm{H}^{0}-\mathrm{T} \Delta \mathrm{S}^{0}
$$

On the other hand, the heat capacity $\left(\Delta C_{p}-p\right.$ subscript indicates that the system is at constant pressure-) of a reaction predicts the change of $\Delta \mathrm{H}^{0}$ and $\Delta \mathrm{S}^{0}$ with temperature and can be expressed as:

$$
\Delta \mathrm{C}_{\mathrm{p}}=\frac{\Delta \mathrm{H}_{\mathrm{T} 2}^{0}-\Delta \mathrm{H}_{\mathrm{T} 1}^{0}}{\mathrm{~T}_{2}-\mathrm{T}_{1}}
$$

or

$$
\Delta \mathrm{C}_{\mathrm{p}}=\frac{\Delta S^{0} \mathrm{~T}_{2}-\Delta S^{0} \mathrm{~T} 1}{\ln \frac{\mathrm{T}_{2}}{\mathrm{~T}_{1}}}
$$

In an ITC experiment a constant temperature is set, a precise amount of ligand is added to a known target molecule concentration and the heat difference is measured between reference and sample cells. To eliminate heats of mixing effects, the ligand and target as well as the reference cell contain identical buffer composition. Subsequent injections of ligand are done until no further heat of binding is observed (all sites are then bound with ligand molecules). The remaining heat generated now comes from dilution of ligand into the target solution. Data should be corrected for the heat of dilution. The heat of binding calculated for every injection is plotted versus the molar ratio of ligand to protein. $\mathrm{K}_{\mathrm{a}}$ is related to the curve shape and binding capacity (n) determined from the ratio of ligand to target at the equivalence point of the curve. Data must be fitted to a binding model. The type of binding must be known from other experimental techniques. Here, we will study the simplest model with a single site. Equations 6 and 7 can be rearranged to find the following relation between $\mathrm{v}$ and $\mathrm{K}_{\mathrm{a}}$ :

$$
\mathrm{K}_{\mathrm{a}}=\frac{\mathrm{v}}{(1-\mathrm{v})[\mathrm{L}]}
$$

Total ligand concentration is known and can be represented as (remember that we are assuming $\mathrm{m}=\mathrm{n}=1)$ :

$$
[\mathrm{L}]_{\text {total }}=[\mathrm{L}]+\mathrm{v}[\mathrm{P}]_{\text {total }}
$$

Combining equations 33 and 34 gives:

$$
\mathrm{v}^{2}-\left(\frac{[\mathrm{L}]_{\text {total }}}{[\mathrm{P}]_{\text {total }}}+\frac{1}{\mathrm{~K}_{\mathrm{a}}[\mathrm{P}]_{\text {total }}}+1\right) \mathrm{v}+\frac{[\mathrm{L}]_{\text {total }}}{[\mathrm{P}]_{\text {total }}}=0
$$

Solving for $\mathrm{v}$ :

$$
\mathrm{v}=\frac{1}{2}\left[\left(\frac{[\mathrm{L}]_{\text {total }}}{[\mathrm{P}]_{\text {total }}}+\frac{1}{\mathrm{~K}_{\mathrm{a}}[\mathrm{P}]_{\text {total }}}+1\right)-\sqrt{\left(\frac{[\mathrm{L}]_{\text {total }}}{[\mathrm{P}]_{\text {total }}}+\frac{1}{\mathrm{~K}_{\mathrm{a}}[\mathrm{P}]_{\text {total }}}+1\right)^{2}-\frac{4[\mathrm{~L}]_{\text {total }}}{[\mathrm{P}]_{\text {total }}}}\right]
$$

The total heat content $(\mathrm{Q})$ in the sample cell at volume $(\mathrm{V})$ can be defined as:

$$
\mathrm{Q}=[\mathrm{PL}] \Delta \mathrm{H}^{0} \mathrm{~V}=\mathrm{v}[\mathrm{P}]_{\text {total }} \Delta \mathrm{H}^{0} \mathrm{~V}
$$


where $\Delta \mathrm{H}^{0}$ is the heat of binding of the ligand to its target. Substituing equation 36 into 37 yields:

$$
\mathrm{Q}=\frac{[\mathrm{P}]_{\text {total }} \Delta \mathrm{H}^{0} \mathrm{~V}}{2}\left[\left(\frac{[\mathrm{L}]_{\text {total }}}{[\mathrm{P}]_{\text {total }}}+\frac{1}{\mathrm{~K}_{\mathrm{a}}[\mathrm{P}]_{\text {total }}}+1\right)-\sqrt{\left(\frac{[\mathrm{L}]_{\text {total }}}{[\mathrm{P}]_{\text {total }}}+\frac{1}{\mathrm{~K}_{\mathrm{a}}[\mathrm{P}]_{\text {total }}}+1\right)^{2}-\frac{4[\mathrm{~L}]_{\text {total }}}{[\mathrm{P}]_{\text {total }}}}\right]
$$

Therefore $\mathrm{Q}$ is a function of $\mathrm{K}_{\mathrm{a}}$ and $\Delta \mathrm{H}^{0}$ (and $\mathrm{n}$, but here we considered it as 1 for simplicity) since $[\mathrm{P}]_{\text {total, }}[\mathrm{L}]_{\text {total }}$ and $\mathrm{V}$ are known for each experiment.

\subsection{Optical spectroscopy}

The goal to be able to determine binding affinity is to measure the equilibrium concentration of the species implied over a range of concentrations of one of the reactants ( $\mathrm{P}$ or $\mathrm{L}$ ). Measuring one of them should be sufficient as total concentrations are known and therefore the others can be calculated by difference from total concentrations and measured equilibrium concentration of one of the species. Plotting the concentration of the complex (PL) against the free concentration of the varying reactant, the binding constant could be calculated.

\subsubsection{Absorbance}

As an example a 1:1 stoichiometry model will be shown, wherein the Lambert-Beer law is obeyed by all the reactants implied. To use this technique we should ensured that the complex (PL) has a significantly different absorption spectrum than the target molecule (P) and a wavelenght at which both molar extinction coefficients are different should be selected. At these conditions the absorbance of the target molecule in the absence of ligand will be:

$$
\mathrm{Abs}_{0}=\varepsilon_{\mathrm{P}} 1[\mathrm{P}]_{\text {total }}
$$

If ligand is added to a fixed total target concentration, the absorbance of the mix can be written as:

$$
\mathrm{Abs}_{\text {mix }}=\varepsilon_{\mathrm{P}} 1[\mathrm{P}]+\varepsilon_{\mathrm{L}} 1[\mathrm{~L}]+\varepsilon_{\mathrm{PL}} 1[\mathrm{PL}]
$$

Since $[\mathrm{P}]_{\text {total }}=[\mathrm{P}]+[\mathrm{PL}]$ and $[\mathrm{L}]_{\text {total }}=[\mathrm{L}]+[\mathrm{PL}]$, equation 40 can be rewritten as:

$$
\mathrm{Abs}_{\text {mix }}=\varepsilon_{\mathrm{P}} 1[\mathrm{P}]_{\text {total }}+\varepsilon_{\mathrm{L}} 1[\mathrm{~L}]_{\text {total }}+\Delta \varepsilon 1[\mathrm{PL}]
$$

where $\Delta \varepsilon=\varepsilon_{\mathrm{PL}}-\varepsilon_{\mathrm{P}}-\varepsilon_{\mathrm{L}}$. If the blank solution against which samples are measured contains $[\mathrm{L}]_{\text {total }}$, then the observed absorbance would be:

$$
\mathrm{Abs}_{\mathrm{obs}}=\varepsilon_{\mathrm{P}} 1[\mathrm{P}]_{\text {total }}+\Delta \varepsilon 1[\mathrm{PL}]
$$

Substracting equation 39 from 42 and incorporating $\mathrm{K}_{\mathrm{a}}$ (equation 5):

$$
\Delta \mathrm{Abs}=\mathrm{K}_{\mathrm{a}} \Delta \varepsilon \mathrm{l}[\mathrm{P}][\mathrm{L}]
$$

$[\mathrm{P}]_{\text {total }}$ can be written as $[\mathrm{P}]_{\text {total }}=[\mathrm{P}]\left(1+\mathrm{K}_{\mathrm{a}}[\mathrm{L}]\right)$ which included in equation 43 yields:

$$
\frac{\Delta \mathrm{Abs}}{\mathrm{l}}=\frac{[\mathrm{P}]_{\text {total }} \mathrm{K}_{\mathrm{a}} \Delta \varepsilon[\mathrm{L}]}{1+\mathrm{K}_{\mathrm{a}}[\mathrm{L}]}
$$


which is the direct plot expressed in terms of spectrophotometric observation. Note that the dependence of $\Delta \mathrm{Abs} / 1$ on [L] is the same as the one shown in equation 7 .

The free ligand concentration is actually unknown. The known concentrations are $[\mathrm{P}]_{\text {total }}$ to which a known $[\mathrm{L}]_{\text {total }}$ is added. In a similar way as shown above for $[\mathrm{P}]_{\text {total, }}[\mathrm{L}]_{\text {total }}$ can be written as:

$$
[\mathrm{L}]_{\text {total }}=[\mathrm{L}] \frac{[\mathrm{P}]_{\text {total }} \mathrm{K}_{\mathrm{a}}[\mathrm{L}]}{1+\mathrm{K}_{\mathrm{a}}[\mathrm{L}]}
$$

From equations 44 and 45 a complete description of the system is obtained. If $[\mathrm{L}]_{\text {total }}$ $>>[\mathrm{P}]_{\text {total }}$ we will have that $[\mathrm{L}]_{\text {total }} \approx[\mathrm{L}]$ from equation 45 , equation 44 can be then analysed with this approximation. With this first rough estimate of $K_{a}$, equation 45 can be solved for the $[\mathrm{L}]$ value for each $[\mathrm{L}]_{\text {total }}$. These values can be used in equation 44 to obtain an improved estimation of $K_{a}$, and this process should be repeated until the solution for $K_{a}$ reaches $a$ constant value. Equation 44 can be solved graphically using any of the plots presented in section 3.1.

\subsubsection{Fluorescence}

Fluorescence spectroscopy is a widely used tool in biochemistry due to its ease, sensitivity to local environmental changes and ability to describe target-ligand interactions qualitatively and quantitatively in equilibrium conditions. In this technique the fluorophore molecule senses changes in its local environment. To analyse ligand-target interactions it is possible to take advantage of the nature of ligands, excepcionally we can find molecules which are essentially non or weakely fluorescent in solution but show intense fluorescence upon binding to their targets (that is the case, for example, of colchicines and some of its analogues). Fluorescence moieties such as fluorescein can be also attached to naturally non-fluorescent ligands to make used of these methods. The fluorescent dye may influence the binding, so an essential control with any tagged molecule is a competition experiment with the untagged molecule. Finally, in a few favourable cases the intrinsic tryptophan fluorescence of a protein changes when a ligand binds, usually decreasing (fluorescence quenching). Again, increasing concentrations of ligand to a fixed concentration of target (or vice versa) are incubated at controlled temperature and fluorescence changes measured until saturation is reached. Binding constant can be determined by fitting data according to equation 11 (Scatchard plot). From fluorescence data (F), $\mathrm{v}$ can be calculated from the relantionship:

$$
\mathrm{v}=\frac{\mathrm{F}_{\max }-\mathrm{F}}{\mathrm{F}_{\max }}
$$

If free ligand has an appreciable fluorescence as compared to ligand bound to its target, then the fluorescence enhancement factor $(Q)$ should be determined. $Q$ is defined as (Mas \& Colman 1985):

$$
\mathrm{Q}=\frac{\mathrm{F}_{\text {bound }}}{\mathrm{F}_{\text {free }}}-1
$$

To determine it, a reverse titration should be done. The enhancement factor can be obtained from the intercept of linear plot of $1 /\left(\left(\mathrm{F} / \mathrm{F}_{0}\right)-1\right)$ against $1 / \mathrm{P}$, where $\mathrm{F}$ and $\mathrm{F}_{0}$ are the observed fluorescence in the presence and absence of target, respectively. Once it is known, the concentration of complex can be determine from a fluorescence titration experiment using: 


$$
[\mathrm{PL}]=[\mathrm{L}]_{\text {total }} \frac{\left(\mathrm{F} / \mathrm{F}_{0}\right)-1}{\mathrm{Q}-1}
$$

Thus the binding constant can be determined from the Scatchard plot as described above.

\subsubsection{Fluorescence anisotropy}

Fluorescence anisotropy measures the rotational diffusion of a molecule. The effective size of a ligand bound to its target usually increases enormously, thus restricting its motion considerably. Changes in anisotropy are proportional to the fraction of ligand bound to its target. Using suitable polarizers at both sides of the sample cuvette, this property can be measured. In a tritation experiment similar to the ones described above, the fraction of ligand bound $\left(\mathrm{X}_{\mathrm{L}}=[\mathrm{PL}] /[\mathrm{L}]_{\text {total }}\right)$ is determined from:

$$
X_{\mathrm{L}}=\frac{\mathrm{r}-\mathrm{r}_{0}}{\mathrm{r}_{\max }-\mathrm{r}_{0}}
$$

where $r$ is the anisotropy of ligand in the presence of the target molecule, $r_{0}$ is the anisotropy of ligand in the absence of target and $r_{\max }$ is the anisotropy of ligand fully bound to its target (note that equation 49 can be used only in the case where ligand fluorescence intensity does not change, otherwise appropriate corrections should be done, see (Lakowicz 1999)). [P] can be calculated from:

$$
[\mathrm{P}]=[\mathrm{P}]_{\text {total }}-\mathrm{X}_{\mathrm{L}}[\mathrm{L}]_{\text {total }}
$$

The binding constant can be determined from the hyperbola:

$$
X_{L}=\frac{K_{a}[P]}{1+K_{a}[P]}
$$

\subsection{Competition methods}

The characterization of a ligand binding let us determine the binding constant of any other ligand competing for the same binding site. Measurements of ligand $(\mathrm{L})$, target $(\mathrm{P})$, reference ligand (R) and both complexes (PR and $\mathrm{PL}$ ) concentrations in the equilibrium permit the calculation of the binding constant $\left(\mathrm{K}_{\mathrm{L}}\right)$ from equation 53 (see below) as the binding constant of the reference ligand $\left(\mathrm{K}_{\mathrm{R}}\right)$ is already known.

$$
\begin{gathered}
\mathrm{L}+\mathrm{R}+\mathrm{P} \leftrightarrow \mathrm{PL}+\mathrm{PR} \\
\mathrm{K}_{\mathrm{L}}=\mathrm{K}_{\mathrm{R}} \frac{[\mathrm{PL}][\mathrm{R}]}{[\mathrm{L}][\mathrm{PR}]}
\end{gathered}
$$

In the case that the reference ligand has been characterized due to the change of a ligand physical property (i.e. fluorescence, absorbance, anisotropy) upon binding, would permit us also following the displacement of this reference ligand from its site by competition with a ligand "blind“ to this signal (Diaz \& Buey 2007). In this kind of experiment equimolar concentrations of the reference ligand and the target molecule are incubated, increasing concentrations of the problem ligand added and the appropiate signal measured. It is possible then to determine the concentration of ligand at which half the reference ligand is bound to its site $\left(\mathrm{EC}_{50}\right)$. Thus $\mathrm{K}_{\mathrm{L}}$ is calculated from:

$$
\mathrm{K}_{\mathrm{L}}=\frac{1+[\mathrm{R}] \mathrm{K}_{\mathrm{R}}}{\mathrm{EC}_{50}}
$$




\section{Drug optimization}

Microtubule stabilizing agents (MSA) comprise a class of drugs that bind to microtubules and stabilize them against disassembly. During the last years, several of these compounds have been approved as anticancer agents or submitted to clinical trials. That is the case of taxanes (paclitaxel, docetaxel) or epothilones (ixabepilone) as well as discodermolide (reviewed in (Zhao et al. 2009)). Nevertheless, anticancer chemotherapy has still unsatisfactory clinical results, being one of the major reasons for it the development of drug resistance in treated patients (Kavallaris 2010). Thus one interesting issue in this field is drug optimization with the aim of improving the potential for their use in clinics: minimizing side-effects, overcoming resistances or enhancing their potency.

Our group has studied the influence of different chemical modifications on taxane and epothilone scaffolds in their binding affinities and the consequently modifications in ligand properties like citotoxicity. The results from these studies firmly suggest thermodynamic parameters as key clues for drug optimization.

\subsection{Epothilones}

Epothilones are one of the most promising natural products discovered with paclitaxel-like activity. Their advantages come from the fact that they can be produced in large amounts by fermentation (epothilones are secondary metabolites from the myxobacterium Sorangiun celulosum), their higher solubility in water, their simplicity in molecular architecture which makes possible their total synthesis and production of many analogs, and their effectiveness against multi-drug resistant cells due to they are worse substrates for P-glycoprotein.

The structure affinity-relationship of a group of chemically modified epothilones was studied. Epothilones derivatives with several modifications in positions C12 and C13 and the side chain in $\mathrm{C} 15$ were used in this work.

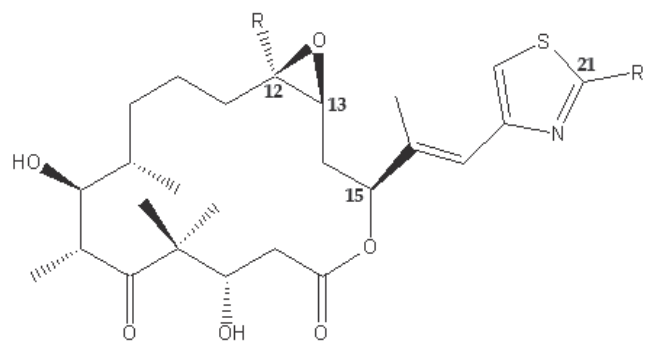

Fig. 1. Epothilone atom numbering.

Epothilone binding affinities to microtubules were measured by displacement of Flutax-2, a fluorescent taxoid probe (fluorescein tagged paclitaxel). Both epothilones A and B binding constants were determined by direct sedimentation which further validates Flutax-2 displacement method.

All compounds studied are related by a series of single group modifications. The measurement of the binding affinity of such a series can be a good approximation of the incremental binding energy provided by each group. Binding free energies are easily calculated from binding constants applying equation 29 . The incremental free energies $\left(\Delta \mathrm{G}^{0}\right)$ change associated with the modification of ligand $\mathrm{L}$ into ligand $\mathrm{S}$ is defined as: 


$$
\Delta \Delta \mathrm{G}^{0}(\mathrm{~L} \rightarrow \mathrm{S})=\Delta \mathrm{G}^{0}(\mathrm{~L})-\Delta \mathrm{G}^{0}(\mathrm{~S})
$$

These incremental binding energies were calculated for a collection of 20 different epothilones as reported in (Buey et al. 2004).

\begin{tabular}{|c|c|c|c|}
\hline Site & Modification & Compounds & $\Delta \Delta \mathrm{G}$ \\
\hline \multirow[t]{7}{*}{ C15 } & $S \rightarrow R$ & $4 \rightarrow 17$ & $\sim 27$ \\
\hline & & $7 \rightarrow 18$ & $\sim 27$ \\
\hline & & $14 \rightarrow 16$ & $17.8 \pm 0.3$ \\
\hline & Thiazole $\rightarrow$ Pyridine & $5 \rightarrow 7$ & $-2.9 \pm 0.2$ \\
\hline & & $6 \rightarrow 8$ & $-2.1 \pm 0.3$ \\
\hline & & $14 \rightarrow 4$ & $-0.2 \pm 0.4$ \\
\hline & & $16 \rightarrow 17$ & $\sim 9.4$ \\
\hline \multirow[t]{6}{*}{$\mathrm{C} 21$} & Methyl $\rightarrow$ Thiomethyl & $2 \rightarrow 3$ & $-2.8 \pm 0.8$ \\
\hline & & $5 \rightarrow 10$ & $-5.9 \pm 0.6$ \\
\hline & & $6 \rightarrow 11$ & $-3.6 \pm 0.3$ \\
\hline & & $8 \rightarrow 12$ & $2.6 \pm 0.3$ \\
\hline & Methyl $\rightarrow$ Hydroxymethyl & $8 \rightarrow 9$ & $1.4 \pm 0.3$ \\
\hline & 5-Thiomethyl-pyridine $\rightarrow$ 6-Thiomethyl-pyridine & $12 \rightarrow 13$ & $4.1 \pm 0.5$ \\
\hline \multirow[t]{13}{*}{$\mathrm{C} 12$} & $S \rightarrow R$ & $4 \rightarrow 7$ & $-2.1 \pm 0.3$ \\
\hline & & $14 \rightarrow 5$ & $0.6 \pm 0.3$ \\
\hline & & $17 \rightarrow 18$ & $\sim-2$ \\
\hline & & $19 \rightarrow 11$ & $9.0 \pm 0.6$ \\
\hline & & $20 \rightarrow 8$ & $1.9 \pm 0.4$ \\
\hline & Epoxide $\rightarrow$ Cyclopropyl & $1 \rightarrow 14$ & $-4.7 \pm 0.4$ \\
\hline & & $3 \rightarrow 19$ & $-5.4 \pm 0.8$ \\
\hline & Cyclopropyl $\rightarrow$ Cyclobutyl & $5 \rightarrow 15$ & $4.1 \pm 0.2$ \\
\hline & S H $\rightarrow$ Methyl & $1 \rightarrow 2$ & $-8.1 \pm 0.6$ \\
\hline & & $4 \rightarrow 20$ & $-1.8 \pm 0.5$ \\
\hline & $R \mathrm{H} \rightarrow$ Methyl & $5 \rightarrow 6$ & $0.4 \pm 0.3$ \\
\hline & & $7 \rightarrow 8$ & $1.2 \pm 0.2$ \\
\hline & & $10 \rightarrow 11$ & $2.7 \pm 0.7$ \\
\hline
\end{tabular}

Table 1. Incremental binding energies of epothilone analogs to microtubules. ( $\Delta \Delta \mathrm{G}$ in $\mathrm{kJ} / \mathrm{mol}$ at $35^{\circ} \mathrm{C}$ ). Data from (Buey et al. 2004).

The data in table 1 show that the incremental binding free energy changes of single modifications give a good estimation of the binding energy provided by each group. Moreover, the effect of the modifications is accumulative, resulting the epothilone derivative with the most favourable modifications (a thiomethyl group at C21 of the thiazole side chain, a methyl group at C12 in the $S$ configuration, a pyridine side chain with $\mathrm{C} 15$ in the $S$ configuration and a cyclopropyl moiety between $\mathrm{C} 12$ and C13) the one with the highest affinity of all the compounds studied $\left(\mathrm{K}_{\mathrm{a}} 2.1 \pm 0.4 \times 10^{10} \mathrm{M}^{-1}\right.$ at $\left.35^{\circ} \mathrm{C}\right)$.

The study of these compounds showed also a correlation between their citotoxic potencial and their affinities to microtubules. The plot of $\log \mathrm{IC}_{50}$ in human ovarian carcinoma cells versus $\log \mathrm{K}_{\mathrm{a}}$ shows a good correlation (figure 2), suggesting binding affinity as an important parameter affecting citotoxicity. 


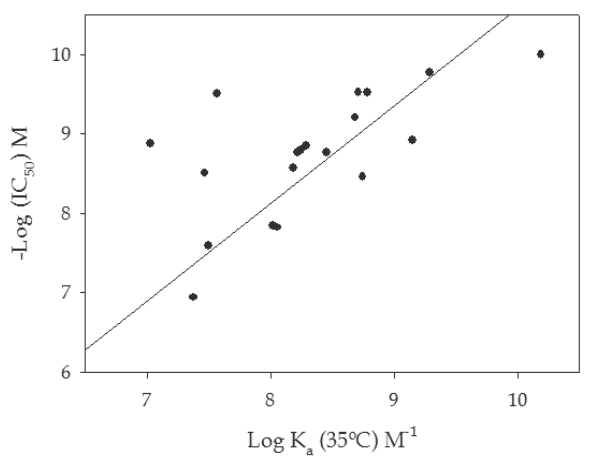

Fig. 2. Dependence of the $\mathrm{IC}_{50}$ of epothilone analogs against $1 \mathrm{~A} 9$ cells on their $\mathrm{K}_{\mathrm{a}}$ to microtubules. Data from (Buey et al. 2004).

\subsection{Taxanes}

Paclitaxel and docetaxel are widely used in the clinics for the treatment of several carcinoma and Kaposi's sarcoma. Nevertheless, their effectiveness is limited due to the development of resistance, beeing its main cause the overexpression and drug efflux activity of transmembrane proteins like P-glycoprotein (Shabbits et al. 2001).

We have studied the thermodynamics of binding of a set of nearly 50 taxanes to crosslinked stabilized microtubules with the aim to quantify the contributions of single modifications at four different locations of the taxane scaffold (C2, C13, C7 and C10).

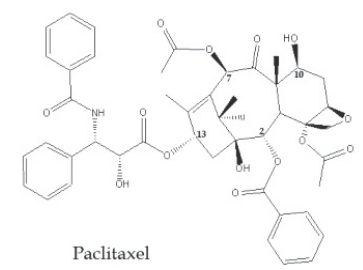

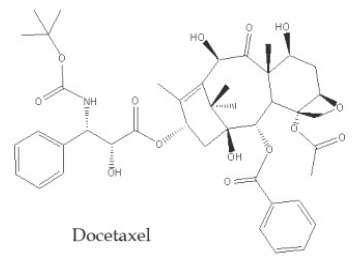

Fig. 3. Taxanes head compounds. Atom numbering

Once confirmed that all the compounds were paclitaxel-like MSA, their affinities were measured using the same competition method mentioned above (section 5.1. displacement of Flutax-2). Seven of the compounds completely displaced Flutax-2 at equimolar concentrations indicating that they have very high affinities and so they are in the limit of the range to be accurately calculated by this method (Diaz\&Buey 2007). The affinities of these compounds were then measured using a direct competition experiment with epothilone- $\mathrm{B}$, a higher-affinity ligand $\left(\mathrm{K}_{\mathrm{a}} 75.0 \times 10^{7}\right.$ at $35^{\circ} \mathrm{C}$ compared with $3.0 \times 10^{7}$ for Flutax-2). With all the binding constants determined at a given temperature, it is possible to determine the changes in binding free energy caused by every single modification as discussed above for epothilones (table 2). 


\begin{tabular}{|c|c|c|c|c|}
\hline Site & Modification & Compounds & $\Delta \Delta \mathrm{G}$ & Average \\
\hline \multirow[t]{37}{*}{$\mathrm{C} 2$} & \multirow[t]{2}{*}{ benzoyl $\rightarrow$ benzylether } & $\mathrm{T} \rightarrow 25$ & 13.2 & $+13.0 \pm 0.2$ \\
\hline & & $21 \rightarrow 24$ & 12.8 & \\
\hline & \multirow[t]{2}{*}{ benzoyl $\rightarrow$ benzylsulphur } & $\mathrm{T} \rightarrow 27$ & 13.6 & $+15.9 \pm 2.3$ \\
\hline & & $21 \rightarrow 26$ & 18.1 & \\
\hline & \multirow[t]{2}{*}{ benzoyl $\rightarrow$ benzylamine } & $\mathrm{T} \rightarrow 38$ & 18.6 & $+20.1 \pm 1.5$ \\
\hline & & $21 \rightarrow 39$ & 21.6 & \\
\hline & \multirow[t]{2}{*}{ benzoyl $\rightarrow$ thiobenzoyl } & $\mathrm{T} \rightarrow 23$ & 19.6 & $+15.9 \pm 3.8$ \\
\hline & & $21 \rightarrow 22$ & 12.1 & \\
\hline & benzoyl $\rightarrow$ benzamide & $21 \rightarrow 42$ & 19.2 & \\
\hline & benzamide $\rightarrow$ 3-methoxy-benzamide & $42 \rightarrow 43$ & -3.4 & \\
\hline & benzamide $\rightarrow$ 3-Cl-benzamide & $42 \rightarrow 44$ & 5.3 & \\
\hline & benzoyl $\rightarrow 3$ methyl- 2 butenoyl & $1 \rightarrow 2$ & 6.2 & \\
\hline & benzoyl $\rightarrow 3$ methyl- 3 butenoyl & $1 \rightarrow 3$ & 4.9 & \\
\hline & benzoyl $\rightarrow$ 2(E)-butenoyl & $1 \rightarrow 9$ & 7.3 & \\
\hline & benzoyl $\rightarrow 3$ methyl- butanoyl & $1 \rightarrow 10$ & 6.3 & \\
\hline & benzoyl $\rightarrow$ 2-debenzoyl-1,2-carbonate & $C \rightarrow 16$ & 5.8 & \\
\hline & \multirow[t]{4}{*}{ benzoyl $\rightarrow$ 3-azido-benzoyl } & $1 \rightarrow 4$ & -8 & $-11.2 \pm 1.3$ \\
\hline & & $\mathrm{T} \rightarrow 12$ & -13.9 & \\
\hline & & $C \rightarrow 14$ & -12.2 & \\
\hline & & $18 \rightarrow 20$ & -10.6 & \\
\hline & \multirow[t]{4}{*}{ benzoyl $\rightarrow$ 3-methoxy-benzoyl } & $1 \rightarrow 5$ & -6.2 & $-7.2 \pm 0.6$ \\
\hline & & $\mathrm{T} \rightarrow 11$ & -8.3 & \\
\hline & & $C \rightarrow 13$ & -8.1 & \\
\hline & & $18 \rightarrow 19$ & -6.3 & \\
\hline & benzoyl $\rightarrow$ 3-Cl-benzoyl & $1 \rightarrow 6$ & -3.1 & \\
\hline & benzoyl $\rightarrow$ 3-Br-benzoyl & $1 \rightarrow 34$ & -2.3 & \\
\hline & benzoyl $\rightarrow$ 3-I-benzoyl & $1 \rightarrow 30$ & -3.3 & \\
\hline & benzoyl $\rightarrow$ 3-ciano-benzoyl & $1 \rightarrow 7$ & 0.6 & \\
\hline & benzoyl $\rightarrow$ 3-methyl-benzoyl & $1 \rightarrow 8$ & 0 & \\
\hline & benzoyl $\rightarrow$ 3-hydroxymethyl-benzoyl & $1 \rightarrow 36$ & 7.2 & \\
\hline & benzoyl $\rightarrow$ 3-hydroxy-benzoyl & $18 \rightarrow 37$ & 9.2 & \\
\hline & 3-Cl-benzoyl $\rightarrow$ 2,4-di-Cl-benzoyl & $6 \rightarrow 29$ & 4.8 & \\
\hline & benzoyl $\rightarrow$ 2,4-di-F-benzoyl & $1 \rightarrow 28$ & 2.7 & \\
\hline & 3-methoxy-benzoyl $\rightarrow$ 2,5-di-methoxy-benzoyl & $5 \rightarrow 35$ & 4.6 & \\
\hline & benzoyl $\rightarrow$ 2-thienoyl & $1 \rightarrow 31$ & 4.1 & \\
\hline & benzoyl $\rightarrow$ 3-thienoyl & $1 \rightarrow 32$ & 1.8 & \\
\hline & benzoyl $\rightarrow 6$-carboxy-pyran-2-one & $1 \rightarrow 41$ & 8.1 & \\
\hline \multirow[t]{6}{*}{$\mathrm{C} 13$} & \multirow[t]{4}{*}{ paclitaxel $\rightarrow$ cephalomannine } & $\mathrm{T} \rightarrow \mathrm{C}$ & 1.9 & $+2.0 \pm 0.2$ \\
\hline & & $11 \rightarrow 13$ & 1.9 & \\
\hline & & $12 \rightarrow 14$ & 1.6 & \\
\hline & & $15 \rightarrow 17$ & 2.4 & \\
\hline & \multirow[t]{2}{*}{ paclitaxel $\rightarrow$ docetaxel } & $23 \rightarrow 22$ & -1.7 & $-3.2 \pm 0.9$ \\
\hline & & $25 \rightarrow 24$ & -6.2 & \\
\hline
\end{tabular}




\begin{tabular}{c|c|c|c|c} 
& & $27 \rightarrow 26$ & -1.3 & \\
& & $38 \rightarrow 39$ & -2.8 & \\
& cephalomannine $\rightarrow$ docetaxel & $\mathrm{T} \rightarrow 21$ & -4.2 & \\
\hline $\mathrm{C} 10$ & $\mathrm{C} \rightarrow 21$ & -3.8 & $-5.6 \pm 1.1$ \\
& acetyl $\rightarrow$ hydroxyl & $17 \rightarrow \mathrm{D}$ & -7.7 & \\
& & $20 \rightarrow 40$ & -5.2 & \\
\hline & propionyl $\rightarrow$ hydroxyl & $\mathrm{T} \rightarrow 15$ & -1.3 & $-1.7 \pm 0.8$ \\
& acetyl $\rightarrow$ propionyl & $\mathrm{C} \rightarrow 17$ & -0.7 & \\
& & $21 \rightarrow \mathrm{D}$ & -3.2 & \\
\hline $\mathrm{C} 7$ & $18 \rightarrow 17$ & 0.9 & \\
& propionyl $\rightarrow$ hydroxyl & $\mathrm{C} \rightarrow 18$ & -1.6 & $-0.5 \pm 0.4$ \\
& $13 \rightarrow 19$ & 0.2 & \\
& & $14 \rightarrow 20$ & 0 & \\
& & $17 \rightarrow 1$ & -1.6 &
\end{tabular}

Table 2. Incremental binding energies of taxane analogs to microtubules. ( $\Delta \Delta \mathrm{G}$ in $\mathrm{kJ} / \mathrm{mol}$ at $35^{\circ} \mathrm{C}$ ). Data from (Matesanz et al. 2008).

In this way, it is possible to select the most favourable substituents at the positions studied and design optimized taxanes. According to the data obtained, the optimal taxane should have the docetaxel side chain at $\mathrm{C} 13$, a 3-N - -benzoyl at $\mathrm{C} 2$, a propionyl at $\mathrm{C} 10$, and a hydroxyl at C7. From compound 1 with a binding energy of $-39.4 \mathrm{~kJ} / \mathrm{mol}$, the modifications selected would increase the binding affinity in $-5.6 \mathrm{~kJ} / \mathrm{mol}$ from the change of the cephalomannine side chain at $\mathrm{C} 13$ to the docetaxel one, $-11.2 \mathrm{~kJ} / \mathrm{mol}$ from the introduction of 3-N $\mathrm{N}_{3}$-benzoyl instead of benzoyl at $\mathrm{C} 2,-1.6 \mathrm{~kJ} / \mathrm{mol}$ from the substitution of a propionyl at $\mathrm{C} 7$ with a hydroxyl, and $-0.9 \mathrm{~kJ} / \mathrm{mol}$ from the change of a hydroxyl at C10 to a propionyl. Thus, this optimal taxane would have a predicted $\Delta \mathrm{G}$ at $35^{\circ} \mathrm{C}$ of $-58.7 \mathrm{~kJ} / \mathrm{mol}$. This molecule was synthesized (compound 40) and its binding affinity measured using the epothilone-B displacement method and the value obtained is in good corespondence with the predicted one: $\mathrm{K}_{\mathrm{a}}=6.28 \pm 0.15 \times 10^{9} \mathrm{M}^{-1} ; \Delta \mathrm{G}=-57.7 \pm 0.1 \mathrm{~kJ} / \mathrm{mol}$ (Matesanz et al. 2008). This value means a 500-fold increment over the paclitaxel affinity.

It is also possible to check the influence of the modifications on the cytotoxic activity determining the $\mathrm{IC}_{50}$ of each compound in the human ovarian carcinoma cells A2780 and their MDR counterparts (A2780AD). The plots of $\log \mathrm{IC}_{50}$ versus $\log \mathrm{K}_{\mathrm{a}}$ (figure 4) indicate that, as in the case of epothilones, both magnitudes are related, and the binding affinity acts as a good predictor of citotoxicity. In this type of MDR cells the high-affinity drugs are circa 100 -fold more cytotoxic than the clinically used taxanes (paclitaxel and docetaxel) and exhibit very low resistance indexes.

The plot of $\log$ resistance index against $\log K_{a}$ shows a bell-shaped curve (figure 5). Resistance index present a maximum for taxanes with similar affinities for microtubules and P-glycoprotein, then rapidly decreases when the affinity for microtubules either increases or decreases. To find an explanation for this behaviour we should note that the intracellular free concentration of the high-affinity compounds will be low. To be pumped out by Pglycoprotein ligands must first bind it, so ligand outflow will decrease with lower free ligand concentrations (discussed in (Matesanz et al. 2008)). In the case of the low-affinity drugs, the concentrations needed to exert their citotoxicity are so high that the pump gets saturated and cannot effectively reduced the intracellular free ligand concentration. 


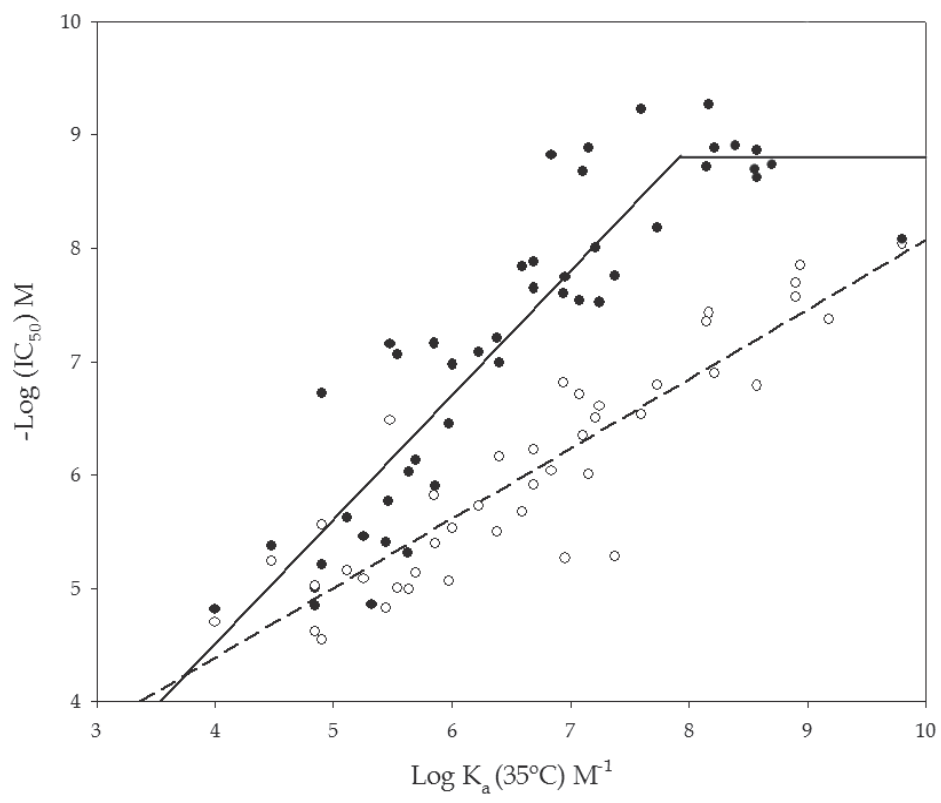

Fig. 4. Dependence of the $\mathrm{IC}_{50}$ of taxane analogs against $\mathrm{A} 2780$ non-resistant cells (black circles, solid line) and A2780AD resistant cells (white circles, dashed line) on their $\mathrm{K}_{\mathrm{a}}$ to microtubules. Data from (Matesanz et al. 2008).

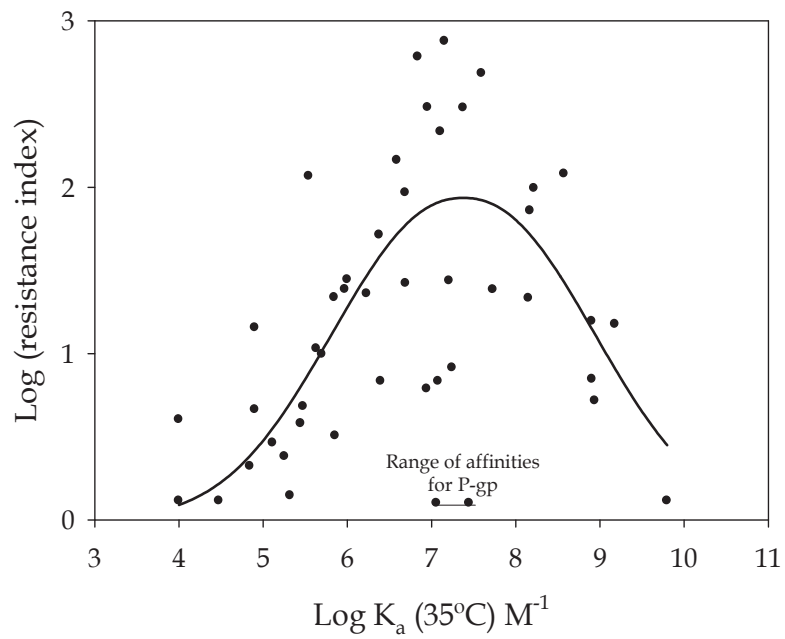

Fig. 5. Dependence of the resistance index of the A2780AD MDR cells on the $\mathrm{K}_{\mathrm{a}}$ of the taxanes to microtubules. Data from (Yang et al. 2007; Matesanz et al. 2008). 


\section{Conclusion}

We found a correlation between binding affinities of paclitaxel-like MSA to microtubules and their citotoxicities in tumoral cells both MDR and non-resistant. The results with taxanes further validate the binding affinity approach as a tool to be used in drug optimization as it was previously discuss for the case of epothilones. Moreover, from the thermodynamic data we could design novel high-affinity taxanes with the ability to overcome resistance in P-glycoprotein overexpressing cells. Anyway, there is a limit concentration below which MSA are not able to kill cells (discussed in (Matesanz et al. 2008)), the highest-affinity compounds studied have no dramatically better citotoxicities than paclitaxel or docetaxel have. Thus, the goal is not to find the drug with the highest cytotoxicity possible but rather to find one able to overcome resistances. The study of taxanes indicates that increased drug affinity could be an improvement in this direction. The extreme example of that come from the covalent binding of cyclostreptin (Buey et al. 2007) (that might be consider as infinite affinity) having a resistance index close to one.

However, in the case of chemically diverse paclitaxel-like MSA, the inhibition of cell proliferation correlates better with enthalpy change than with binding constants (Buey et al. 2005) suggesting that favourable enthalpic contributions to the binding are important to improve drug activity as it has been shown for statins and HIV protease inhibitors (Freire 2008).

\section{References}

Alberts, B., D. Bray, J. Lewis, M. Raff, K. Roberts\&J. D. Watson, Eds. (1994). Molecular Biology of the Cell. New York, Garland Science.

Benesi, H. A.\&J. H. Hildebrand (1949). "A Spectrophotometric Investigation of the Interaction of Iodine with Aromatic Hydrocarbons." journal of the american chemical society 71(8): 2703-2707.

Buey, R. M., I. Barasoain, E. Jackson, A. Meyer, P. Giannakakou, I. Paterson, S. Mooberry, J. M. Andreu\&J. F. Diaz (2005). "Microtubule interactions with chemically diverse stabilizing agents: thermodynamics of binding to the paclitaxel site predicts cytotoxicity." Chem Biol 12(12): 1269-1279.

Buey, R. M., E. Calvo, I. Barasoain, O. Pineda, M. C. Edler, R. Matesanz, G. Cerezo, C. D. Vanderwal, B. W. Day, E. J. Sorensen, J. A. Lopez, J. M. Andreu, E. Hamel\&J. F. Diaz (2007). "Cyclostreptin binds covalently to microtubule pores and lumenal taxoid binding sites." Nat Chem Biol 3(2): 117-125.

Buey, R. M., J. F. Diaz, J. M. Andreu, A. O'Brate, P. Giannakakou, K. C. Nicolaou, P. K. Sasmal, A. Ritzen\&K. Namoto (2004). "Interaction of epothilone analogs with the paclitaxel binding site: relationship between binding affinity, microtubule stabilization, and cytotoxicity." Chem Biol 11(2): 225-236.

Connors, K. A., Ed. (1987). Binding Constants: The Measurement of Molecular Complex Stability. New York, wiley-interscience.

Connors, K. A.\&S. Mecozzi, Eds. (2010). Thermodynamics of Pharmaceutical Systems. An Introduction to Theory and Applications. new york, wiley-intersciences.

Diaz, J. F.\&R. M. Buey (2007). "Characterizing ligand-microtubule binding by competition methods." Methods Mol Med 137: 245-260. 
Freire, E. (2008). "Do enthalpy and entropy distinguish first in class from best in class?" Drug Discovery Today 13(19-20): 869-874.

Hill, A. V. (1910). "The possible effects of the aggregation of the molecules of haemoglobin on its dissociation curves." The Journal of Physiology 40(Suppl): iv-vii.

Hirose, K. (2001). "A Practical Guide for the Determination of Binding Constants." Journal of Inclusion Phenomena and Macrocyclic Chemistry 39(3): 193-209.

Ingham, K. C. (1975). "On the application of Job's method of continuous variation to the stoichiometry of protein-ligand complexes." Analytical Biochemistry 68(2): 660-663.

Job, P. (1928). "Formation and stability of inorganic complexes in solution." Annali di Chimica 9: 113-203.

Johnson, I. S., H. F. Wright, G. H. Svoboda\&J. Vlantis (1960). "Antitumor principles derived from Vinca rosea Linn. I. Vincaleukoblastine and leurosine." Cancer Res 20: 10161022.

Kavallaris, M. (2010). "Microtubules and resistance to tubulin-binding agents." Nat Rev Cancer 10(3): 194-204.

Lakowicz, J. R. (1999). Principles of fluorescence spectroscopy. New York, Kluwer Academic/ Plenum Publishers.

Mas, M. T.\&R. F. Colman (1985). "Spectroscopic studies of the interactions of coenzymes and coenzyme fragments with pig heart oxidized triphosphopyridine nucleotide specific isocitrate dehydrogenase." Biochemistry 24(7): 1634-1646.

Matesanz, R., I. Barasoain, C. G. Yang, L. Wang, X. Li, C. de Ines, C. Coderch, F. Gago, J. J. Barbero, J. M. Andreu, W. S. Fang\&J. F. Diaz (2008). "Optimization of taxane binding to microtubules: binding affinity dissection and incremental construction of a high-affinity analog of paclitaxel." Chem Biol 15(6): 573-585.

Ohtaka, H.\&E. Freire (2005). "Adaptive inhibitors of the HIV-1 protease." Progress in Biophysics and Molecular Biology 88(2): 193-208.

Ohtaka, H., S. Muzammil, A. Schön, A. Velazquez-Campoy, S. Vega\&E. Freire (2004). "Thermodynamic rules for the design of high affinity HIV-1 protease inhibitors with adaptability to mutations and high selectivity towards unwanted targets." The International Journal of Biochemistry \& Cell Biology 36(9): 1787-1799.

Pollard, T. D. (2010). "A Guide to Simple and Informative Binding Assays." Mol. Biol. Cell 21(23): 4061-4067.

Ruben, A. J., Y. Kiso\&E. Freire (2006). "Overcoming Roadblocks in Lead Optimization: A Thermodynamic Perspective." Chemical Biology \& Drug Design 67(1): 2-4.

Scatchard, G. (1949). "The attractions of proteins for small molecules and ions." Annals of the New York Academy of Sciences 51(4): 660-672.

Shabbits, J. A., R. Krishna\&L. D. Mayer (2001). "Molecular and pharmacological strategies to overcome multidrug resistance." Expert Rev Anticancer Ther 1(4): 585-594.

Yang, C. G., I. Barasoain, X. Li, R. Matesanz, R. Liu, F. J. Sharom, D. L. Yin, J. F. Diaz\&W. S. Fang (2007). "Overcoming Tumor Drug Resistance with High-Affinity Taxanes: A SAR Study of C2-Modified 7-Acyl-10-Deacetyl Cephalomannines." ChemMedChem 2(5): 691-701.

Zhao, Y., W.-S. Fang\&K. Pors (2009). "Microtubule stabilising agents for cancer chemotherapy." Expert Opinion on Therapeutic Patents 19(5): 607-622. 
(C) 2011 The Author(s). Licensee IntechOpen. This is an open access article distributed under the terms of the Creative Commons Attribution 3.0 License, which permits unrestricted use, distribution, and reproduction in any medium, provided the original work is properly cited. 